\title{
Licochalcone B Exhibits Anti-inflammatory Effects via Modulation of NF- $\kappa B$ and AP-1
}

\author{
Jin-Kyung Kim ${ }^{1, \uparrow}$ and Jong-Gab Jun ${ }^{2}$ \\ ${ }^{I}$ Department of Biomedical Science, Catholic University of Daegu, Gyeongsan-Si 38430, Korea \\ ${ }^{2}$ Department of Chemistry and Institute of Natural Medicine, Hallym University, Chuncheon 24252, Korea
}

The present study investigated the mechanisms of licochalcone B (LicB)-mediated inhibition of the inflammatory response in murine macrophages. RAW264.7 murine macrophages were cultured in the absence or presence of lipopolysacharide (LPS) with LicB. LicB suppressed the generation of nitric oxide and the pro-inflammatory cytokines interleukin (IL)-1 $\beta$, IL- 6 and tumor necrosis factor- $\alpha$. LicB also inhibited the expression of mRNA for inducible nitric oxide synthase and pro-inflammatory cytokines induced by LPS. Moreover, LicB inhibited nuclear factor- $\mathrm{KB}$ (NF- $\mathrm{KB})$ and activator protein-1 translocation into the nucleus in a dose-dependent manner. Thus, LicB mainly exerts its antiinflammatory effects by inhibiting the LPS-induced NF- $\mathrm{kB}$ and activator protein-1 signaling pathways in macrophages, which subsequently diminishes the expression and release of various inflammatory mediators. LicB shows promise as a therapeutic agent in inflammatory diseases.

Key Words: Inflammation, NF-kB, Macrophage, LPS, Licorice

\section{INTRODUCTION}

Inflammation is a protective response of host defense against injury or infection, and begins the healing process. However, when inflammation becomes chronic, it is harmful and may lead to a host of diseases, such as arthritis, atherosclerosis, and even cancer (Libby et al., 2002; Hartman and Frishman, 2014; Guina et al., 2015). Among cells that regulate inflammatory responses, macrophages are important in the host defensive system against microbial infections through growth suppression (Gilroy and Maeyer, 2015; Smith et al., 2015). Macrophages generate and release several

*Received: November 20, 2015 / Revised: December 17, 2015

Accepted: December 24, 2015

${ }^{\dagger}$ Corresponding author: Jin-Kyung Kim. Department of Biomedical Science, College of Natural Science, Catholic University of Daegu, 13-13 Hayang-RO, Gyeoungsan-Si 38430, Korea.

Tel: +82-53-850-3774, Fax: +82-53-850-3774

e-mail: toto0818@cu.ac.kr

(C)The Korean Society for Biomedical Laboratory Sciences. All rights reserved. inflammatory mediators that include nitric oxide (NO), prostaglandins, interleukin (IL)-1 $\beta$, IL-6 and tumor necrosis factor-alpha $(\mathrm{TNF}-\alpha)$ in response to regulating agents like lipopolysaccharide (LPS) or other microbial agents. Toll-like receptors (TLRs) play key roles in helping to recognize microbial insults with subsequent immune responses in macrophages (Dinarello, 2011; Chun et al., 2012; Arango and Descoteaux, 2014). LPS activates TLR4 and induces two signaling pathways: the myeloid differentiation primary response protein 88-dependent and Toll-like/IL-1 receptorcontaining adapter inducing interferon- $\beta$ (TRIF)-dependent signaling pathways (Kawai and Akira, 2006). The first pathway induces the activation of mitogen-activated protein kinases (MAPKs) and nuclear factor- $\mathrm{kB}$ (NF- $\mathrm{\kappa B})$, leading to the release of pro-inflammatory cytokines including IL-1 $\beta$, IL-6 and TNF- $\alpha$ (Kawai and Akira, 2006; O'Neill and Bowie, 2007). The second pathway activates the downstream of TRIF kinases, leading to the phosphorylation and activation of interferon regulatory factor 3 and the consequent expression 
of type I interferons (Kawai and Akira, 2006; O'Neill and Bowie, 2007).

Botanical dietary supplements are popularly used as natural alternatives to traditional therapy and often possess a variety of anti-inflammatory, antioxidant and chemopreventive activities (Balunas and Kinghorn, 2005; Snelten et al., 2012; Di Lorenzo et al., 2013). They provide extraordinarily diverse chemical scaffolds on which chemical modification can be applied to recognize the structural requirements that are responsible for the related biological activity and to derive new lead drug molecules with superior activity. In addition, an increasing number of studies also indicate that diets rich in anti-inflammatory phytochemicals may have beneficial effects in ameliorating metabolic syndrome, a constellation of abnormal cardiometabolic factors that increase risk of cardiovascular disease and type 2 diabetes (Agarwal et al., 2012; Xiao and Högger, 2015). However, the mechanisms by which such beneficial effects are mediated are not well understood.

Licorice root from Glycyrrhiza inflate has been used in traditional and herbal medicines. This species contains unusual phenolic compounds, called retrochalcones, which include licochalcone (Lic) A to E, and echinatin (Kao et al., 2014). In our previous screening for anti-inflammatory small molecule compounds, we identified LicB (Fig. 1A) and demonstrated its' suppression of NO production (Kim et al., 2014). However, whether it regulates LPS-induced inflammatory signaling and whether it could be a potential antiinflammatory agent remain unknown. In the present study, we investigated the anti-inflammatory properties of LicB in response to LPS, and explored the underlying mechanisms involved in its actions.

\section{MATERIALS AND METHODS}

\section{Chemicals and reagents}

LicB was synthesized as previously described (Kim et al., 2014). LPS derived from Escherichia coli and dimethylsulfoxide (DMSO) were obtained from Sigma-Aldrich (St. Louis, MO, USA). Dulbecco's modified Eagle's medium (DMEM), fetal bovine serum (FBS), penicillin, and streptomycin were obtained from Hyclone (Logan, UT, USA).<smiles>COc1c(/C=C/C(=O)c2ccc(O)cc2)ccc(O)c1O</smiles>

B

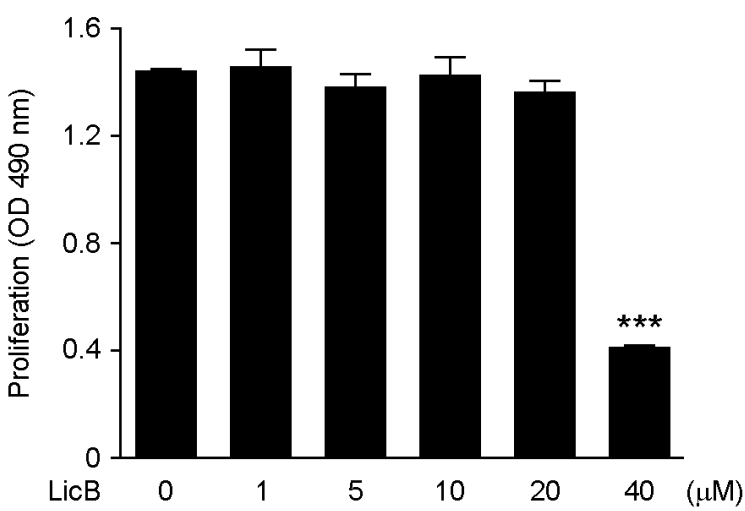

Fig. 1. Chemical structure of LicB (A) and effects of LicB on murine macrophage viability (B). RAW264.7 cells were treated with the indicated concentrations of LicB for $24 \mathrm{~h}$ and proliferation was determined as described in Materials and Methods. The results are reported as mean \pm SEM of three independent experiments done in triplicate. Statistical significance is based on the difference when compared with $0 \mu \mathrm{M}$-treated cells $(* * * P<0.001)$.

The final concentrations of DMSO never exceeded $0.1 \%$, which did not affect the assay systems. The rabbit polyclonal antibodies (Abs) used were anti-inducible NO synthase (iNOS), anti-phospho-c-Jun N-terminal kinase (JNK), antiextracellular signal-regulated kinase (ERK1/2), anti-phosphop38 rabbit polyclonal, anti-p38, anti-poly ADP ribose polymerase (PARP), anti-NFkB-p65, anti-c-Fos and anti-c-Jun (all from Cell Signaling Technology, Danvers, MA, USA). Monoclonal Abs (mAbs) used were anti- $\beta$-actin (Sigma-

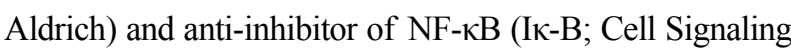
Technology).

\section{Cell culture and cell proliferation assay}

RAW264.7 murine macrophages obtained from the Korean Cell Bank (Seoul, Korea) were cultured in DMEM containing 10\% FBS, $100 \mathrm{U} / \mathrm{ml}$ penicillin and $100 \mu \mathrm{g} / \mathrm{ml}$ streptomycin at $37{ }^{\circ} \mathrm{C}$ in $5 \% \mathrm{CO}_{2}$. The effect of $\mathrm{LicB}$ on cell proliferation was tested using the CellTiter $96^{\circledR} \mathrm{AQ}_{\text {ueous }}$ One 
Solution Assay of cell proliferation (Promega, Madison, WI, USA), which uses colorimetry to count the number of viable cells. RAW264.7 cells were plated at a density of $2 \times 10^{4}$ cells in a 96-well flat-bottom plate. LicB was added to each plate at indicated concentrations. After $24 \mathrm{~h}$ incubation, the number of viable cells was counted according to the manufacturer's instructions. The assay is based on the reduction of 3-(4,5-dimethylthiazol-2-yl)-5-(3-carboxymethoxyphenyl) -2-(4-sulfophenyl)-2H-tetrazolium (MTS) to formazan. The quantity of the product in the cell culture is indicated by the optical density of formazan at $490 \mathrm{~nm}$, which is directly proportional to the number of living cells.

\section{Measurement of nitrite}

The amount of nitrite produced by the mouse macrophages was measured in the RAW264.7 cell culture supernatant. RAW264.7 cells were plated at a density of $2.5 \times 10^{5}$ cells in a 48-well cell culture plate with $500 \mu$ of culture medium and incubated for $12 \mathrm{~h}$. They were then treated with indicated concentrations of LicB plus LPS (500 ng/ml) and incubated for another $24 \mathrm{~h}$. The amount of nitrite produced was measured using the Griess reagent system (Promega).

\section{Cytokine measurement}

The amount of IL-1 $\beta$, IL- 6 and TNF- $\alpha$ in the cell culture supernatant was measured using an ELISA kit (eBioscience, San Diego, CA, USA). RAW264.7 cells were plated in a 48 -well cell culture plate at a density of $2.5 \times 10^{5}$ cells and incubated with indicated concentrations of LicB in 500 $\mathrm{ng} / \mathrm{ml}$ LPS for $24 \mathrm{~h}$. The culture supernatant was collected and assayed according to the manufacturer's instructions to determine the amount of IL- $1 \beta$, IL- 6 and TNF- $\alpha$ released from the cells.

\section{Quantitative real-time reverse-transcription polymerase chain reaction ( $q R T-P C R)$}

Total RNA was isolated from RAW264.7 cells using Trizol Reagent (Invitrogen, Carlsbad, CA, USA). DNA was eliminated from total RNA using RNA Qualified RNase-Free DNase (Promega) and cDNA was synthesized by GoScript ${ }^{\mathrm{TM}}$ Reverse Transcription System (Promega). qRT-PCR assay was carried out with LightCycler (Roche Diagnostics, Basel,
Switzerland) using LightCycler FastStart DNA Master SYBR Green I (Roche Diagnostics). All the experiments were repeated twice in triplicate. Transcripts of glyceraldehyde3-phosphate dehydrogenase (GAPDH) as a housekeeping gene were quantified as endogenous RNA of reference to normalize each sample. Relative quantities were estimated by the $-\Delta \Delta \mathrm{Ct}$ method. The primers used in this study corresponded to mouse iNOS: F 5'-CCT CCT CCA CCC TAC CAA GT-3', R 5'-CAC CCA AAG TGC TTC AGT CA-3', mouse IL-1 $\beta$ : F 5'-TGT CTC AGC CTC TTC TCA TT-3', R 5'-AGA TGA TCT GAG TGT GAG GG-3', mouse IL-6: F 5'-CAT CCA GTT GCC TTC TTG GGA-3', R 5'-CTG AAG GAC TCT GGC TTG TC-3', mouse TNF- $\alpha$ : F 5'TGT CTC AGC CTC TTC TCA TT-3' R 5'-AGA TGA TCT GAG TGT GAG GG-3' and mouse GAPDH: F 5'-TCT TGC TCA GTG TCC TTG C-3'. R 5'-CTT TGT CAA GCT CAT TTC CTG G-3'

\section{Western blotting}

Whole cell and nuclear extracts (30 $\mu$ g protein/lane) were separated by $10 \%$ sodium dodecyl sulfate-polyacrylamide gel electrophoresis (SDS-PAGE). The separated proteins were electrophoretically transferred to nitrocellulose membranes. The membranes were incubated with the indicated Abs and specific bands were visualized using an enhanced chemiluminescence kit (Amersham Biosciences, Piscataway, NJ, USA).

\section{Statistical analyses}

The data were presented means \pm SEM. The values were evaluated by one-way ANOVA with Bonferroni multiple comparison range tests using GraphPad Prism 4.0 software (GraphPad Software Inc, San Diego, CA). Statistically significant differences were considered at a $P$-value $<0.05$.

\section{RESULTS}

\section{Effect of LicB on RAW264.7 macrophage viability}

To evaluate the effect of LicB on the proliferation of RAW264.7 macrophages, we applied various concentrations of LicB and performed a MTS assay. LicB had an obvious cytotoxic effect at $40 \mu \mathrm{M}$, but had no cytotoxic effect at 


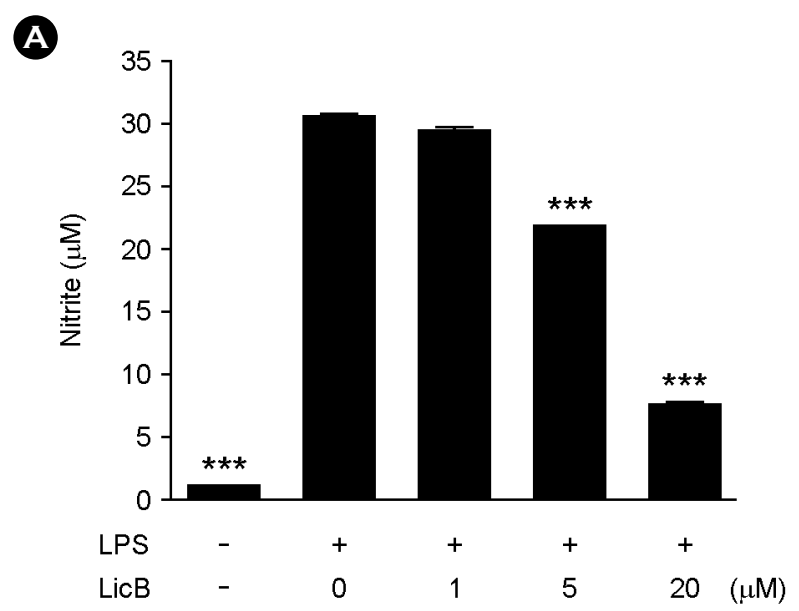

B

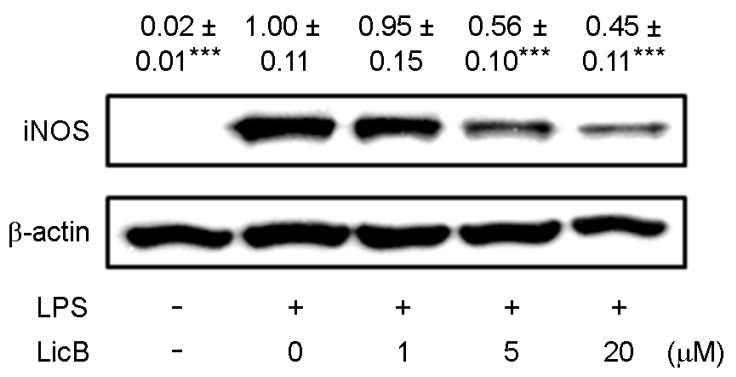

Fig. 2. Effects of LicB on LPS-induced NO release and iNOS expression. (A) RAW264.7 cells were treated with $0 \sim 20 \mu \mathrm{M}$ of LicB in the presence of $500 \mathrm{ng} / \mathrm{ml}$ of LPS or with LPS alone for $24 \mathrm{~h}$, and NO release were determined. (B) Thirty micrograms of protein obtained from each cell lysate was resolved by $10 \%$ SDSPAGE for iNOS determination. $\beta$-actin expression is shown as a loading control. The bands were quantified using NIH image analysis software and their relative intensity was expressed as fold-change against the image of the LPS-stimulated RAW264.7 cells. The results are reported as mean \pm SEM of three independent experiments. Statistical significance is based on the difference when compared with LPS-stimulated cells $(* * * P<0.001)$.

$\leq 20 \mu \mathrm{M}$ (Fig. 1B). Concentrations of 1,5 and $20 \mu \mathrm{M}$ were used for further exploration of the anti-inflammatory activity of LicB.

\section{LicB inhibits NO release and iNOS expression}

Since the release of NO by macrophages is a hallmark inflammatory reaction, we first measured NO levels in LPS-stimulated RAW264.7 cells to investigate the antiinflammatory effect of LicB. LPS stimulation led to a significant increase in the levels of NO in the culture supernatant
(Fig. 2A). Treating the RAW264.7 cells with LicB inhibited LPS-induced generation of NO in a concentration-dependant manner. The nitrite concentrations in LPS-stimulated RAW264.7 cells, in cells exposed to 5 and $20 \mu \mathrm{M} \mathrm{LicB}$ were 30.5 $\pm 0.2 \mu \mathrm{M}, 21.2 \pm 0.1 \mu \mathrm{M}$ and $8.2 \pm 0.2 \mu \mathrm{M}$ respectively. Consistent with the findings related to NO production, the protein expression of iNOS induced by LPS in RAW264.7 cells was also reduced significantly by LicB treatment $(5$ and $20 \mu \mathrm{M}$; Fig. 2B). This indicated that the LicB-induced reduction in iNOS expression is responsible for the inhibition of NO production.

LicB suppresses pro-inflammatory cytokine production in LPS-stimulated RAW 264.7 cells

Other pro-inflammatory cytokines play important roles in various inflammatory diseases in addition to NO. So, we also examined whether $\mathrm{LicB}$ regulates production of major pro-inflammatory cytokines, IL- $1 \beta$, IL- 6 and TNF- $\alpha$. To assess the inhibitory effects of LicB on LPS-induced production of pro-inflammatory cytokines, ELISA was performed on supernatants after $24 \mathrm{~h}$ in the presence of LicB. The release of these cytokines was scarcely detectable in resting RAW 264.7 macrophages, whereas large amounts of IL-1 $\beta$, IL- 6 and TNF- $\alpha$ were induced upon exposure to LPS alone (Fig. 3). Addition of LicB (5 and $20 \mu \mathrm{M}$ ) to the cells significantly decreased the LPS-induced release of these pro-inflammatory cytokines in a concentration-dependent manner.

LicB down-regulates expression of inflammatory mediators at the transcriptional level in LPS-stimulated RAW264.7 cells

Next, we determined whether the changes in protein levels of iNOS and pro-inflammatory cytokines in LicB-treated RAW264.7 cells was caused by the alternations of their mRNA levels. RAW264.7 cells were stimulated for $8 \mathrm{~h}$ with LPS in the presence of increasing concentrations of LicB, and qRT-PCR analysis was performed. LicB treatment decreased mRNA levels of iNOS, IL-1 $\beta$, IL-6 and TNF- $\alpha$ in a concentration-dependent manner (Fig. 4), suggesting that LicB could suppress LPS-induced iNOS, IL-1 $\beta$, IL-6 and TNF- $\alpha$ expression at the transcriptional level and protein level. 
(A)

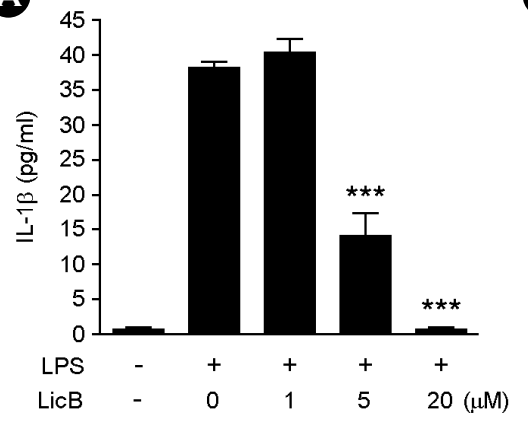

B

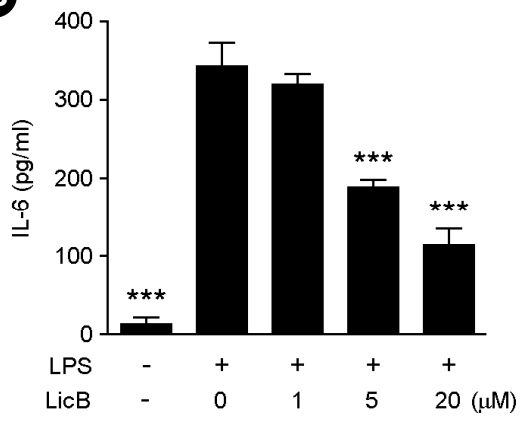

C

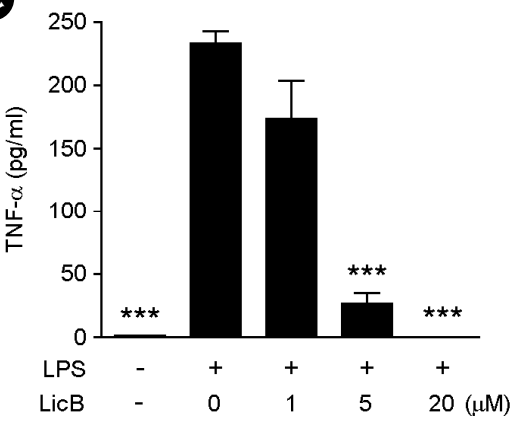

Fig. 3. Effects of LicB on LPS-induced pro-inflammatory cytokine production in murine macrophages. RAW264.7 cells were treated with $0 \sim 20 \mu \mathrm{M} \mathrm{LicB}$ in the presence of $500 \mathrm{ng} / \mathrm{ml}$ LPS or with LPS alone for $24 \mathrm{~h}$. The cell culture medium was collected and the amount of (A) IL-1 $\beta$, (B) IL-6 and (C) TNF- $\alpha$ released was measured as described in Materials and Methods. The results are reported as mean \pm SEM of three independent experiments in triplicate. Statistical significance is based on the difference when compared with LPS-stimulated cells $(* * * P<0.001)$

A

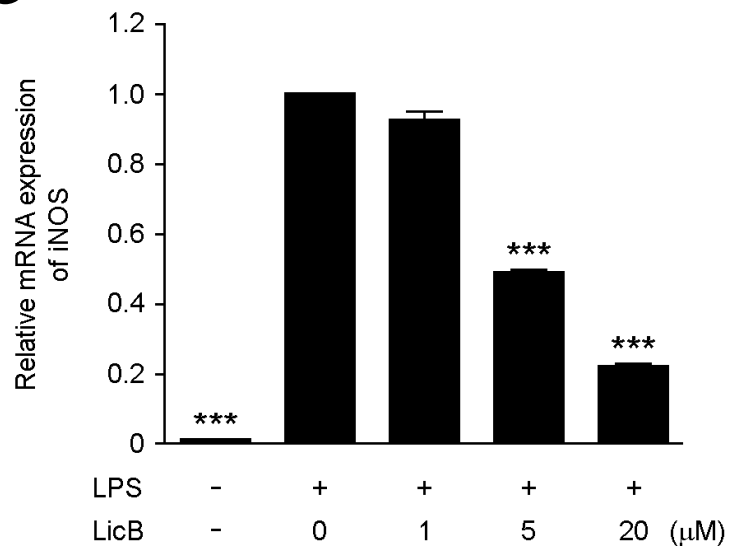

B

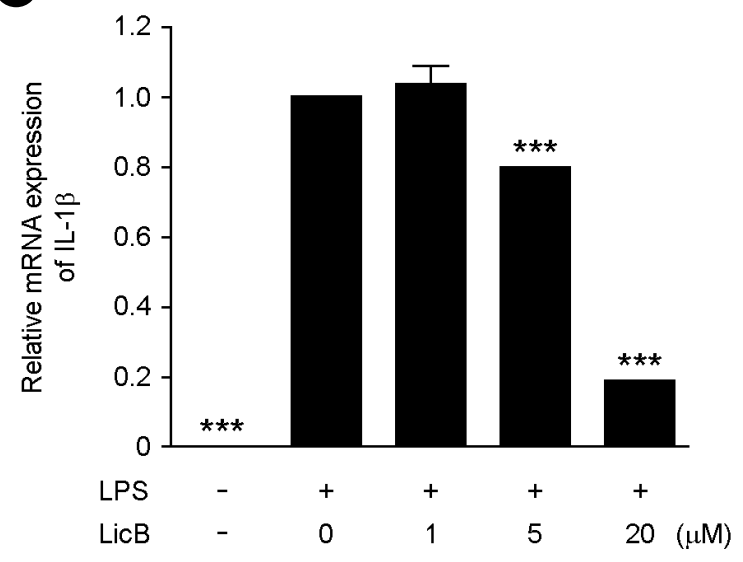

C

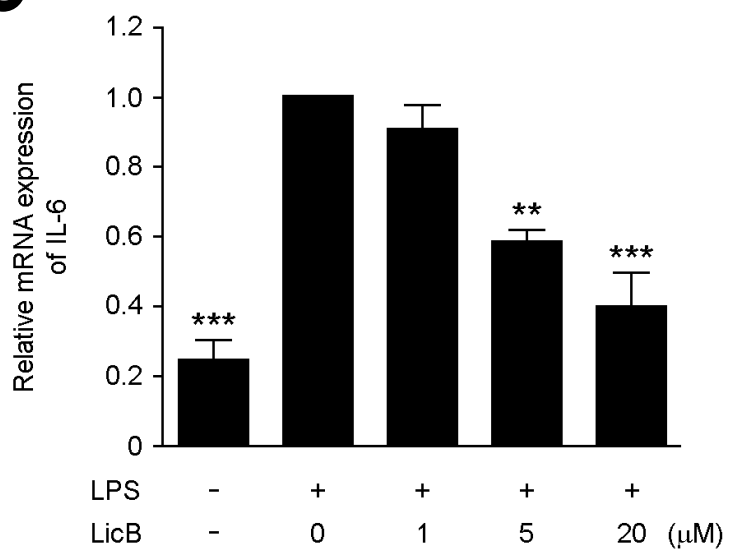

D

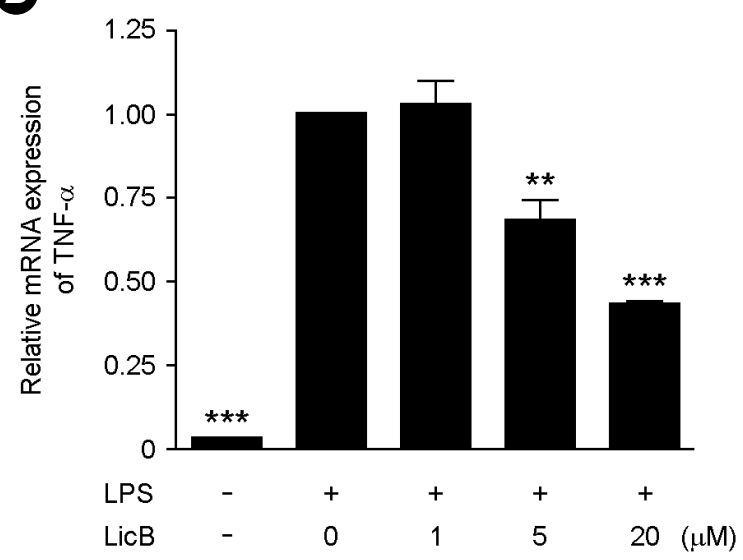

Fig. 4. Effect of LicB on LPS-induced mRNA expression of inflammatory mediators. RAW264.7 cells were treated with the indicated concentrations of $\mathrm{LicB}$ in the presence of $500 \mathrm{ng} / \mathrm{ml}$ LPS or with LPS alone for $6 \mathrm{~h}$. Quantification of mRNA levels was performed using real-time RT-PCR described in Materials and Methods. The histogram showing the fold difference in (A) iNOS (B) IL-1 (C) IL-6 and (D) TNF- $\alpha$ mRNA levels was normalized to GAPDH. Each value represents the mean \pm SEM of three separate experiments. Statistical significance is based on the difference when compared with LPS-stimulated cells $(* * P<0.01, * * * P<0.001)$. 
(A)

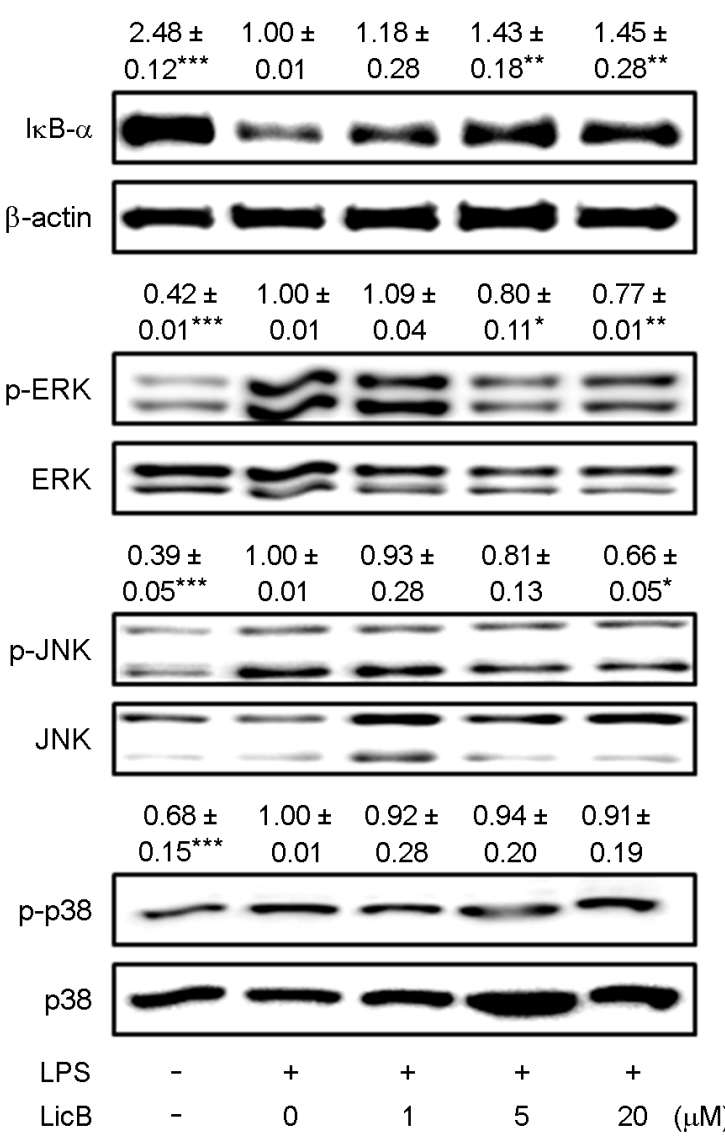

B
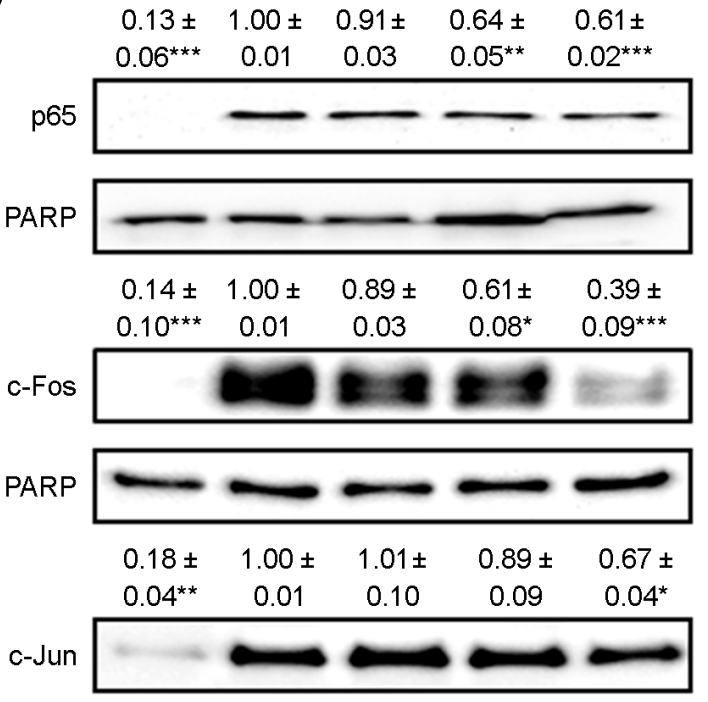

PARP

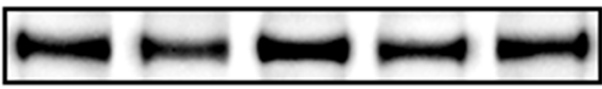

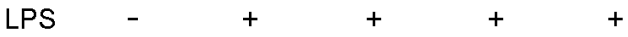

$\begin{array}{lllllll}\text { LicB } & - & 0 & 1 & 5 & 20\end{array}$

Fig. 5. Effect of LicB on LPS-induced NF-אB and MAPK activation. (A) RAW264.7 cells were plated in 100-mm dishes. After $12 \mathrm{~h}$ of seeding, cells were treated with different doses of LicB in the presence of LPS (500 ng/ml) for $20 \mathrm{~min}$. Whole cell extracts were immunoblotted with the indicated Abs. $\beta$-actin was used as a control. (B) RAW264.7 cells treated with LPS alone or with various concentrations of LicB in the presence of LPS for $1 \mathrm{~h}$. Nuclear protein was prepared and subjected to Western blot analysis by using antibodies specific for indicates Abs and equivalent loading of nuclear protein was determined by reprobing the blots with anti-PARP. The bands were quantified using NIH image analysis software and their relative intensity was expressed as fold against the image of the LPS-stimulated RAW264.7 cells. The results are reported as mean \pm SEM of three independent experiments. Statistical significance is based on the difference when compared with LPS-stimulated cells $(* P<0.05$, $* * P<0.01$, $* * * P<0.001)$.

\section{LicB-reduced inflammatory mediator release is regulated by NF-KB and MAPK}

$\mathrm{NF}-\kappa \mathrm{B}$ is a key transcription factor that mediates proinflammatory responses (Pasparakis, 2009; Baker et al., 2011). To better demonstrate the mechanisms involved in the inhibition of inflammatory mediators gene expression, we sought to determine whether LicB could suppress LPSinduced degradation of $\mathrm{I} \kappa \mathrm{B}$, which leads to the activation of NF- $\mathrm{KB}$ and its translocation to the nucleus. As shown in

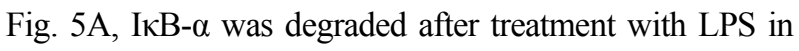
RAW264.7 cells. However, co-treatment with LicB at 5 and
$20 \mu \mathrm{M}$ markedly inhibited LPS-induced degradation of I $\kappa \mathrm{B}-\alpha$, thereby preventing the translocation of $\mathrm{p} 65$ into the nucleus. LPS-induced nuclear translocation of p65 was suppressed by co-treating cells with LPS and LicB (Fig. 5B).

LPS-induced MAPK signaling pathways, such as the ERK, p38, and JNK pathways, play main roles in TLR4-mediated pro-inflammatory cytokines production (Kaminska, 2005). We, therefore, evaluated the regulatory effects of LicB on LPS-induced MAPK activity by profiling the expression of phospho-MAPKs. LicB markedly inhibited LPS-induced phosphorylation of ERK and JNK, while phosphorylation of p38 was unchanged by treatment with LicB (Fig. 5A). 
Since MAPKs mainly regulate AP-1, we investigated if LicB exerts anti-inflammatory activities by affecting AP-1 activity. Nuclear translocation of c-Fos and c-Jun, a subunit of AP-1, was inhibited by LicB treatment in a dose-dependent manner (Fig. 5B). Taken together, these results demonstrated that exogenous addition of LicB in the culture medium suppresses LPS-induced inflammatory mediators by inhibiting the activation of NF- $\mathrm{KB}$ and MAPKs.

\section{DISCUSSION}

Licochalcone is a molecule contained in licorice root (Radix Glycyrrhizae) extract. The root is obtained from perennial plants native to Mediterranean countries, central to southern Russia, and certain regions of Asia. Glycyrrhiza glabra L. and Glycyrrhiza uralensis Fisch. (Fam. Leguminosae) roots are the commonest sources of licorice used in cosmetics, foods, tobacco and in both traditional and herbal medicines. Licorice contains several classes of secondary metabolites that have been associated with numerous human health benefits. Licorice and its bioactive ingredients such as glycyrrhizin, glabridin, licochalcone A, licoricidin and licorisoflavan A possess potential beneficial effects in oral diseases. More specifically, the pharmacological activities of licorice and their effects in the treatment for different human diseases, such as cancer, atherosclerosis, gastric ulcers, hepatitis, bacterial infections and immunodeficiency, have been demonstrated (Isbrucker and Burdock, 2006; Asl and Hosseinzadeh, 2008). The aim of this study was to evaluate the anti-inflammatory effects of LicB in LPS-stimulated RAW264.7 macrophages.

A variety of transcription factors including NF- $\kappa \mathrm{B}$ and AP-1 are involved in the transcriptional regulation of inflammatory mediators including iNOS, TNF- $\alpha$, IL- $1 \beta$ and IL-6 (Kaminska, 2005; Pasparakis, 2009; Baker et al., 2011; Schonthaler et al., 2011). NF- $\mathrm{B}$ transcription factors are pivotal regulators of inflammation and immunity that control expression of important immunoregulatory genes (Pasparakis, 2009; Baker et al., 2011). In unstimulated cells, the inactive $\mathrm{p} 50 / \mathrm{p} 65$ element of NF- $\mathrm{KB}$ is maintained as a heterodimer in the cytoplasm that are bound to $\mathrm{I} \kappa \mathrm{B}$ proteins. I $\mathrm{KB}$ inhibits $\mathrm{NF}-\kappa \mathrm{B}$ activation in the cytoplasm of unstimulated macro- phages. Stimulation of cells with LPS induces IкB degradation, which releases the NF- $\mathrm{B}$ heterodimer leading to the transcription of inflammatory genes like iNOS and inflammatory cytokines, such as IL-1 $\beta$, IL- 6 and TNF- $\alpha$ (Pasparakis, 2009; Baker et al., 2011). The transcription factor NF- $\kappa B$ plays a vital role in the development of immune and inflammatory responses by controlling the expression of inflammation associated with proteins, such as iNOS and inflammatory cytokines (Pasparakis, 2009; Baker et al., 2011). The expression levels of iNOS and inflammatory cytokines are regulated by $\mathrm{NF}-\kappa \mathrm{B}$ activation, which is the major regulator of the transcription of these proteins (Pasparakis, 2009; Baker et al., 2011). These inflammatory mediators play a central role in inflammatory disorders, and their suppression is the major objective of anti-inflammatory therapies. Presently, LicB down-regulated NF-kB pathways and inhibited the expressions of iNOS, IL-1 $\beta$, IL-6 and TNF- $\alpha$. These observations indicate that LicB have beneficial roles in the regulation of inflammation.

Independent or synergistic with NF- $\mathrm{BB}, \mathrm{AP}-1$ is a heterodimeric protein constituted by members of the Jun and Fos families of DNA-binding proteins (Jochum et al., 2001). Both c-Jun and c-Fos are phosphorylated and activated by MAPKs, such as ERK, p38 and JNK kinase (Pulverer et al., 1991; Chen et al., 1996). Many stimuli induce the binding of AP-1 to the promoter region of various genes that govern cellular processes including inflammation, proliferation and apoptosis (Wisdom, 1999). Thus, AP-1 proteins are recognized as regulators of cytokine expression and important modulators in inflammatory diseases (Pulverer et al., 1991; Chen et al., 1996; Wisdom, 1999; Jochum et al., 2001; Zenz et al., 2008; Schonthaler et al., 2011). Presently, LicB inhibited c-Jun and c-Fos activation by blocking the phosphorylation of ERK and JNK.

Taken together, our results demonstrate that LicB inhibits the production of inflammatory mediators in LPS-induced RAW264.7 cells through suppression of the ERK and JNKmediated AP-1 pathway, as well as NF- $\kappa$ B pathwa. Since AP-1 is a critical transcription factor during the process of inflammation, LicB could be an interesting lead compound for novel anti-inflammatory drugs.

LicB might exert a broad range of anti-inflammatory 
effects by controlling the activities of key transcription factors. It is not yet clear whether these seemingly pleiotropic effects of LicB are the result of its control of several individual proteins, such as NF- $\mathrm{BB}$ and AP-1, or of a 'master' upstream factor. Further investigations are warranted to unravel the molecular mechanism underlying the anti-inflammatory activities of LicB and to sort out the complex relationship between the large number of signaling molecules and transcription factors involved in the regulation of inflammation.

\section{Acknowledgements}

This research was supported by research grants from Catholic University of Daegu in 2014.

\section{Conflict of interest}

The authors declare that there are no conflicts of interest.

\section{REFERENCES}

Agarwal M, Parameswari RP, Vasanthi HR, Das DK. Dynamic action of carotenoids in cardioprotection and maintenance of cardiac health. Molecules. 2012. 17: 4755-4769.

Arango DG, Descoteaux A. Macrophage cytokines: involvement in immunity and infectious diseases. Front Immunol. 2014. 8: 319.

Asl MN, Hosseinzadeh H. Review of pharmacological effects of Glycyrrhiza sp. and its bioactive compounds. Phytother Res. 2008. 22: 709-724.

Baker RG, Hayden MS, Ghosh S. NF-אB, inflammation, and metabolic disease. Cell Metab. 2011. 13: 11-22.

Balunas MJ, Kinghorn AD. Drug discovery from medicinal plants. Life Sci. 2005. 78: 431-441.

Blasius AL, Beutler B. Intracellular toll-like receptors. Immunity. 2010. 32: 305-315.

Chen RH, Juo PC, Curran T, Blenis J. Phosphorylation of c-Fos at the $\mathrm{C}$-terminus enhances its transforming activity. Oncogene. 1996. 12: 1493-1502.

Chun J, Choi RJ, Khan S, Lee DS, Kim YC, Nam YJ, Lee DU, Kim YS. Alantolactone suppresses inducible nitric oxide synthase and cyclooxygenase-2 expression by down-regulating NF- $\mathrm{kB}$, MAPK and AP-1 via the MyD88 signaling pathway in LPSactivated RAW 264.7 cells. Int Immunopharmacol. 2012. 14: 375-383.
Di Lorenzo C, Dell'Agli M, Badea M, Dima L, Colombo E, Sangiovanni E, Restani P, Bosisio E. Plant food supplements with anti-inflammatory properties: a systematic review (II). Crit Rev Food Sci Nutr. 2013. 53: 507-516.

Dinarello CA. A clinical perspective of IL-1 $\beta$ as the gatekeeper of inflammation. Eur J Immunol. 2011. 41: 1203-1217.

Gilroy D, De Maeyer R. New insights into the resolution of inflammation. Semin Immunol. 2015. 27: 161-168.

Guina T, Biasi F, Calfapietra S, Nano M, Poli G. Inflammatory and redox reactions in colorectal carcinogenesis. Ann N Y Acad Sci. 2015. 1340: 95-103.

Hartman J, Frishman WH. Inflammation and atherosclerosis: a review of the role of interleukin- 6 in the development of atherosclerosis and the potential for targeted drug therapy. Cardiol Rev. 2014. 22: 147-151.

Isbrucker RA, Burdock GA. Risk and safety assessment on the consumption of Licorice root (Glycyrrhiza sp.), its extract and powder as a food ingredient, with emphasis on the pharmacology and toxicology of glycyrrhizin. Regul Toxicol Pharmacol. 2006. 46: 167-192.

Jochum W, Passegué E, Wagner EF. AP-1 in mouse development and tumorigenesis. Oncogene. 2001. 20: 2401-24112.

Kao TC, Wu CH, Yen GC. Bioactivity and potential health benefits of licorice. J Agric Food Chem. 2014. 62: 542-553.

Kaminska B. MAPK signalling pathways as molecular targets for anti-inflammatory therapy-from molecular mechanisms to therapeutic benefits. Biochim Biophys Acta. 2005. 1754: 253 -262 .

Kawai T, Akira S. TLR signaling. Cell Death Differ. 2006. 13: 816 -825 .

Kim SJ, Kim CG, Yun SR, Kim JK, Jun JG. Synthesis of licochalcone analogues with increased anti-inflammatory activity. Bioorg Med Chem Lett. 2014. 24: 181-185.

Libby P, Ridker PM, Maseri A. Inflammation and atherosclerosis. Circulation. 2002. 105: 1135-1143.

O'Neill LA, Bowie AG. The family of five: TIR-domain-containing adaptors in Toll-like receptor signalling. Nat Rev Immunol. 2007. 7: 353-364.

Pasparakis M. Regulation of tissue homeostasis by NF-kappaB signaling: implications for inflammatory diseases. Nat Rev Immunol. 2009. 9: 778-788.

Pulverer BJ1, Kyriakis JM, Avruch J, Nikolakaki E, Woodgett JR. Phosphorylation of c-jun mediated by MAP kinases. Nature. 1991. 353: 670-674.

Schonthaler HB, Guinea-Viniegra J, Wagner EF. Targeting inflam- 
mation by modulating the Jun/AP-1 pathway. Ann Rheum Dis. 2011. 70 Suppl: 109-112.

Smith AM, Sewell GW, Levine AP, Chew TS, Dunne J, O'Shea NR, Smith PJ, Harrison PJ, Macdonald CM, Bloom SL, Segal AW. Disruption of macrophage pro-inflammatory cytokine release in Crohn's disease is associated with reduced optineurin expression in a subset of patients. Immunology. 2015. 144: 45-55.

Snelten CS, Dietz B, Bolton JL. Modulation of estrogen chemical carcinogenesis by botanical supplements used for postmenopausal women's health. Drug Discov Today Dis Mech. 2012.
9: 47-54.

Wisdom R. AP-1: one switch for many signals. Exp Cell Res. 1999. 253: $180-185$.

Xiao JB, Högger P. Dietary polyphenols and type 2 diabetes: current insights and future perspectives. Curr Med Chem. 2015. 22: 23-38.

Zenz R, Eferl R, Scheinecker C, Redlich K, Smolen J, Schonthaler HB, Kenner L, Tschachler E, Wagner EF. Activator protein 1 (Fos/Jun) functions in inflammatory bone and skin disease. Arthritis Res Ther. 2008. 10: 201. 